\title{
WAVELET DECOMPOSITION OF CALDERÓN-ZYGMUND OPERATORS ON FUNCTION SPACES
}

\author{
KA-SING LAU and LIXIN YAN \\ (Received 18 December 2000; revised 1 March 2003) \\ Communicated by A. H. Dooley
}

\begin{abstract}
We make use of the Beylkin-Coifman-Rokhlin wavelet decomposition algorithm on the CalderónZygmund kernel to obtain some fine estimates on the operator and prove the $T(1)$ theorem on Besov and Triebel-Lizorkin spaces. This extends previous results of Frazier et al., and Han and Hofmann.

2000 Mathematics subject classification: primary 42B20, 46B30.

Keywords and phrases: Calderón-Zygmund operator, Besov space, Triebel-Lizorkin space, wavelet, BCR algorithm.
\end{abstract}

\section{Introduction}

In recent years, there has been significant progress on the problem of boundedness of generalized Calderón-Zygmund operators on various function spaces. The operators in question can be described as follows. Let $K(x, y)$ be a continuous function defined on $\left(\mathbb{R}^{n} \times \mathbb{R}^{n}\right) \backslash\{x=y\}$ and let $T: \mathscr{D} \longrightarrow \mathscr{D}^{\prime}$ be the linear operator associated with the kernel $K(x, y)$, that is,

$$
\langle T \varphi, \psi\rangle=\iint_{\mathbb{R}^{n} \times \mathbb{R}^{n}} K(x, y) \varphi(y) \psi(x) d y d x
$$

where $\varphi, \psi \in \mathscr{D}$ are $C^{\infty}$-test functions on $\mathbb{R}^{n}$ with disjoint supports. For convenience, we write

$$
\Delta K\left(x, x^{\prime} ; y, y^{\prime}\right)=\left|K(x, y)-K\left(x^{\prime}, y^{\prime}\right)\right|+\left|K(y, x)-K\left(y^{\prime}, x^{\prime}\right)\right| .
$$

Both authors are partially supported by a direct grant from the Chinese University of Hong Kong. The second author is also partially supported by NSF of China and NSF of Guangdong Province.

(C) 2004 Australian Mathematical Society 1446-7887/04 \$A2.00+0.00 
It is customary to assume that $K(x, y)$ satisfies the following pointwise conditions:

$$
\begin{gathered}
|K(x, y)| \leq C|x-y|^{-n}, \quad \text { and } \\
\left|\Delta K\left(x, x^{\prime} ; y, y\right)\right| \leq C\left|x^{\prime}-x\right|^{\gamma}|x-y|^{-n-\gamma} \text { for }|x-y| \geq 2\left|x^{\prime}-x\right|,
\end{gathered}
$$

where $0<\gamma \leq 1$.

In their celebrated paper [4], David and Journé characterized the type of kernel $K(x, y)$ for which $T$ is a bounded operator on $L^{2}$. This is now called the $T(1)$ theorem. They proved that under conditions (1.1) and (1.2) on $K(x, y), T$ extends to a bounded operator on $L^{2}$ if and only if both $T(1)$ and $T^{*}(1)$ are BMO functions, and $T$ has the following weak boundedness property (WBP): For $\varphi, \psi \in \mathscr{D}$ with $\operatorname{diam}(\operatorname{supp} \varphi), \operatorname{diam}(\operatorname{supp} \psi) \leq t$,

$$
|\langle T \varphi, \psi\rangle| \leq t^{n}\left(\|\varphi\|_{\infty}+t\|\nabla \varphi\|_{\infty}\right)\left(\|\psi\|_{\infty}+t\|\nabla \psi\|_{\infty}\right) .
$$

Later, Meyer [11] improved the theorem by replacing the pointwise assumption with the following integral assumption on $K(x, y)$ :

$$
\begin{gathered}
\sup _{r>0} \int_{r \leq|x-y|<2 r}(|K(x, y)|+|K(y, x)|) d y \leq C, \quad \text { and } \\
\sum_{k=0}^{\infty}(k+1) B(k)<\infty, \text { with } \\
B(k)=\sup _{\substack{x>0 \\
|u|+|v| \leq r}}\left(\int_{2^{k} r \leq|x-y|<2^{k+1} r} \Delta K(x+u, x ; y+v, y) d y\right) .
\end{gathered}
$$

The $T(1)$ theorem has also been considered by Lemarié on the Besov spaces [10], Frazier $e t$ al on the Triebel-Lizorkin spaces [7], and Han and Hofmann on both classes of spaces [8]. The definitions of such spaces can be stated as follows (see [13]):

Let $\mathscr{S}\left(\mathbb{R}^{n}\right)$ be the space of tempered test functions. Let $\varphi \in \mathscr{S}\left(\mathbb{R}^{n}\right)$ with supp $\hat{\varphi} \subset$ $\left\{\xi \in \mathbb{R}^{n}: 1 / 2 \leq|\xi| \leq 2\right\}$ and $|\hat{\varphi}(\xi)| \geq c>0$ for $3 / 5 \leq|\xi| \leq 5 / 3$; put $\varphi_{j}(x)=$ $2^{j n} \varphi\left(2^{j} x\right)$ and $Q_{j}(f)(x)=\varphi_{j} * f(x)$. For $\alpha \in \mathbb{R}$ and $0<p, q<\infty$, the Besov spaces $\dot{B}_{p}^{\alpha, q}$ is the collection of all $f \in \mathscr{S}^{\prime} / \mathscr{P}$ (the tempered distributions modulo polynomials) satisfying

$$
\|f\|_{\dot{B}_{r}^{\text {a.q }}}=\left(\sum_{j}\left(2^{j \alpha}\left\|Q_{j} f\right\|_{p}\right)^{q}\right)^{1 / q}<\infty .
$$

The Triebel-Lizorkin space is defined analogously, $\dot{F}_{p}^{\alpha, q}$ being the collection of all $f \in \mathscr{S}^{\prime} / \mathscr{P}$ such that

$$
\|f\|_{F_{\nu}^{i \omega, q}}=\left\|\left(\sum_{j}\left(2^{j \alpha}\left|Q_{j} f\right|\right)^{q}\right)^{1 / q}\right\|_{p}<\infty .
$$


In this paper, we will prove the following two theorems:

THEOREM 1.1. Suppose T satisfies the WBP (1.3), the kernel $K(x, y)$ satisfies

(i) $\left(1.1^{\prime}\right)$ and $\left(1.2^{\prime}\right)$. If $T(1)=T^{*}(1)=0$, then $T$ is bounded on $\dot{B}_{p}^{0, q}, 1 \leq p$, $q<\infty$.

(ii) (1.1') and $\sum_{k=0}^{\infty} 2^{k \alpha} B(k)<\infty$. If $T(1)=0$, then $T$ is bounded on $\dot{B}_{p}^{\alpha, q}$, $0<\alpha<1$ and $1 \leq p, q<\infty$.

THEOREM 1.2. Suppose $T$ satisfies the WBP (1.3), the kernel $K(x, y)$ satisfies

(i) $\left(1.1^{\prime}\right)$ and $\sum_{k=0}^{\infty}(k+1)^{2-1 / q} B(k)<\infty$. If $T(1)=T^{*}(1)=0$, then $T$ is bounded on $\dot{F}_{p}^{0, q}, 1 \leq p, q<\infty$.

(ii) (1.1') and $\sum_{k=0}^{\infty} 2^{k \alpha} B(k)<\infty$. If $T(1)=0$, then $T$ is bounded on $\dot{F}_{p}^{\alpha, q}$, $0<\alpha<1$ and $1 \leq p, q<\infty$.

We remark that the two theorems extend the results of Han and Hofmann [8]; they need to assume that $B(k) \leq C 2^{-k \epsilon}$ for $0<\alpha<\epsilon$ in both theorems. For $-1<\alpha<0$, Theorem 1.1 and Theorem 1.2 also hold by interchanging the role of $T(1)$ and $T^{*}(1)$ because of the duality (the dual of $\dot{B}_{p}^{\alpha, q}$ is $\dot{B}_{p^{\prime}}^{-\alpha, q^{\prime}}$ and similarly for $\dot{F}_{p}^{\alpha, q}$ ).

Note that $\dot{F}_{p}^{0.2}$ is of special interest because it is the Hardy space $H^{1}$ when $p=1$ and is $L^{p}$ when $p>1$. For the Hardy space $H^{1}$, the kernel condition in Theorem 1.2 is $\sum_{k}(k+1)^{3 / 2} B(k)<\infty$. In [5], $T$ is proved to be bounded on $L^{2}$ under the kernel condition $\sum_{k}(k+1)^{1 / 2} B(k)<\infty$. By the interpolation theorem, a direct application of the theorem yields the following result, which is stronger than the corresponding case stated in (i).

COROLlary 1.3. Suppose $T$ satisfies the WBP (1.3), the kernel $K(x, y)$ satisfies $\left(1.1^{\prime}\right)$ and $\sum_{k}(k+1)^{1 / 2+2|1 / p-1 / 2|} B(k)<\infty$. If $T(1)=T^{*}(1)=0$, then $T$ is bounded on $L^{p}, 1<p<\infty$.

The main tool used in proving the theorems is wavelets, initiated in [2] and [5]. This is quite different from the approaches in $[7,8,10,11]$. The proof of Theorem 1.1 depends on the Beylkin-Coifman-Rohklin wavelet decomposition of the operator $T$. For Theorem 1.2, we first prove the boundedness of $T$ on $\dot{F}_{1}^{\alpha, q}$ using an atomic decomposition on this space. This, together with an interpolation on $\dot{F}_{p}^{\alpha, p}\left(=\dot{B}_{p}^{\alpha, p}\right)$, yields the boundedness of $T$ for the other case.

The paper is organized as follows. In Section 2 we will give some preliminaries on wavelets and the BCR decomposition of $T$. We also set up the proof in terms of wavelet terminology. The $T(1)$ theorem on the Besov spaces is proved in Section 3 and on the Triebel-Lizorkin spaces in Section 4. 


\section{Preliminaries}

For simplicity, we only consider the one dimensional case. The higher dimensional case is similar.

Let us recall the concept of multiresolution analysis in $L^{2}(\mathbb{R})[12]$ : it is an increasing sequence of closed linear subspaces $\left\{V_{j}\right\}_{j \in \mathbb{Z}} \subseteq L^{2}(\mathbb{R})$ with the following properties:

(i) $\bigcap_{j \in \mathbb{Z}} V_{j}=\{0\}, \bigcup_{j \in \mathbb{Z}} V_{j}$ is dense in $L^{2}(\mathbb{R})$;

(ii) For every $j \in \mathbb{Z}$ and $f \in L^{2}(\mathbb{R}), f \in V_{j} \Longleftrightarrow f(2 \cdot) \in V_{j+1}$;

(iii) There exists a $\varphi$ in $V_{0}$ such that $\varphi(x-k), k \in \mathbb{Z}$, is an orthonormal basis for $V_{0}$.

The above $\varphi$ is called a scaling function. Note that by adjusting a normalization constant, $\sum_{k} \phi(x-k)=1$ for all $x \in \mathbb{R}[3]$. For each $j \in \mathbb{Z}$, we define $\varphi_{j k}(x)=2^{j / 2} \varphi\left(2^{j} x-k\right), k \in \mathbb{Z}$. The sequence $\left\{\varphi_{j k}\right\}_{k \in \mathbb{Z}}$ forms an orthonormal basis for $V_{j}$. From $\varphi$ we can construct a wavelet function $\psi$. Then $\left\{\psi_{j k}\right\}_{k \in \mathbb{Z}}$ forms an orthonormal basis for $W_{j}$, the orthogonal complement to $V_{j}$ inside $V_{j+1}$, that is, $V_{j+1}=V_{j} \oplus W_{j}$. It follows that $\left\{\psi_{j k}\right\}_{j, k \in \mathbb{Z}}$ is an orthonormal basis for $L^{2}(\mathbb{R})$. In this paper, we assume that the wavelets are compactly supported, say $\operatorname{supp} \varphi$, supp $\psi \subseteq[0, M]$ for some integer $M$. Also we assume that they have the desirable degree of smoothness whenever needed.

We need the following characterizations of the Besov and Triebel-Lizorkin spaces $[6,12]$.

PROPOSITION 2.1. Suppose $\psi \in C^{\gamma}$ is a compactly supported wavelet and $\left\{\psi_{j k}\right\}_{j, k \in \mathbb{Z}}$ forms an orthonormal basis of $L^{2}(\mathbb{R})$. Let $f$ be locally integrable and write $f(x)=$ $\sum_{j k} \alpha(j, k) \psi_{j k}(x)$ formally.

(i) For $0<\alpha<\gamma, 1 \leq p, q<\infty, f \in \dot{B}_{p}^{\alpha, q}$ if and only if

$$
\left(\sum_{j}\left(\sum_{k}\left|2^{(-1 / p+\alpha+1 / 2) j} \alpha(j, k)\right|^{p}\right)^{q / p}\right)^{1 / q}<\infty .
$$

(ii) For $0<\alpha<\gamma, 1 \leq p, q<\infty, f \in \dot{F}_{p}^{\alpha, q}$ if and only if

$$
A(f)(x)=\left(\sum_{j, k} 2^{(\alpha+1 / 2) j q}|\alpha(j, k)|^{q} \chi\left(2^{j} x-k\right)\right)^{1 / q} \in L^{p}(\mathbb{R}),
$$

where $\chi$ denotes the characteristic function of $[0,1)$. In this case, $\|f\|_{\dot{F}_{r}{ }^{\alpha, q}} \approx\|A(f)\|_{p}$.

Let $P_{j}: L^{2}(\mathbb{R}) \rightarrow V_{j}$ be the orthonormal projection and $Q_{j}=P_{j+1}-P_{j}$. Then $Q_{j}: L^{2}(\mathbb{R}) \rightarrow W_{j}$ is the corresponding orthonormal projection. In [2], Beylkin, 
Coifman and Rokhlin give a decomposition of $T$ in terms of $P_{j}$ and $Q_{j}$ :

$$
T=\sum_{-\infty}^{\infty} P_{j} T Q_{j}+\sum_{-\infty}^{\infty} Q_{j} T P_{j}+\sum_{-\infty}^{\infty} Q_{j} T Q_{j}
$$

The corresponding distribution kernel is

$$
\begin{aligned}
K(x, y)= & \sum_{j k l} a(j, k, l) \varphi_{j k}(x) \psi_{j l}(y)+\sum_{j k l} b(j, k, l) \psi_{j k}(x) \varphi_{j l}(y) \\
& +\sum_{j k l} c(j, k, l) \psi_{j k}(x) \psi_{j l}(y),
\end{aligned}
$$

where

$$
\begin{aligned}
& a(j, k, l)=\left\langle T \psi_{j l}, \varphi_{j k}\right\rangle=\left\langle K, \varphi_{j k} \otimes \psi_{j l}\right\rangle, \\
& b(j, k, l)=\left\langle T \varphi_{j l}, \psi_{j k}\right\rangle=\left\langle K, \psi_{j k} \otimes \varphi_{j l}\right\rangle, \\
& c(j, k, l)=\left\langle T \psi_{j l}, \psi_{j k}\right\rangle=\left\langle K, \psi_{j k} \otimes \psi_{j l}\right\rangle .
\end{aligned}
$$

We call such $a(j, k, l), b(j, k, l), c(j, k, l)$ the BCR-coefficients.

It is easy to show that

Proposition 2.2. Suppose $T$ satisfies the conditions in Theorem 1.1 or Theorem 1.2, then $T^{*}(1)=0$ implies that for any $j, l \in \mathbb{Z}, \sum_{k} a(j, k, l)=0$; similarly $T(1)=0$ implies that for any $j, k \in \mathbb{Z}, \sum_{l} b(j, k, l)=0$.

PROOF. Assuming that $T^{*}(1)=0$ and using $\sum_{k} \varphi(x-k)=1$, we have

$$
\sum_{k} a(j, k, l)=\sum_{k}\left\langle T \psi_{j l}, \varphi_{j k}\right\rangle=\left\langle T \psi_{j l}, 2^{j / 2}\right\rangle=\left\langle\psi_{j l}, 2^{j / 2} T^{*}(1)\right\rangle=0 .
$$

The second part can be proved similarly.

PROPOSITION 2.3. Suppose T satisfies the conditions in Theorem 1.1 or Theorem 1.2. Let

$$
A(m)=\sup _{j, l}\left\{\sum_{k: 2^{m} \leq|k-l|<2^{m+1}}(|a(j, k, l)|+|a(j, l, k)|+|b(j, k, l)|+|b(j, l, k)|)\right\} .
$$

Then there exists $C$ such that $A(m) \leq C B(m)$ for all $m \geq 0$.

Moreover we have

$$
\sup _{j . l} \sum_{k}(|c(j, k, l)|+|c(j, l, k)|)<\infty .
$$


PRoof. We first observe that $A(m)<\infty$ for each $m \geq 0$. This comes directly from the WBP in (1.3) and the expressions for $a(j, k, l)$ and $b(j, k, l)$. By using this, it suffices to prove the inequality for $2^{m}>M$.

Let $y_{0}=2^{-j} l$, then

$$
\begin{aligned}
a(j, k, l) & =\iint K(x, y) \varphi_{j k}(x) \psi_{j l}(y) d x d y \\
& =2^{j} \iint\left(K\left(x, y+y_{0}\right)-K\left(x, y_{0}\right)\right) \varphi\left(2^{j} x-k\right) \psi\left(2^{j} y\right) d x d y \\
& \leq C \sup _{y \in\left[0,2^{-j} M\right]} \int_{\left[k 2^{-j},(k+M) 2^{-j}\right]}\left|K\left(x, y+y_{0}\right)-K\left(x, y_{0}\right)\right| d x .
\end{aligned}
$$

Hence for $2^{m}>M$,

$$
\sum_{k: 2^{m} \leq|k-l|<2^{m+1}}|a(j, k, l)| \leq C \sup _{\left.y \in \mid 0,2^{-j} M\right]} \int_{E}\left|K\left(x, y+y_{0}\right)-K\left(x, y_{0}\right)\right| d x,
$$

where $E=\left\{x \in \mathbb{R}: 2^{m-j} \leq\left|x-y_{0}\right| \leq 2^{m+1-j}+2^{-j} M\right\}$. According to the definition of $B(m)$, we have $\sum_{k: 2^{m} \leq|k-l|<2^{m+1}}|a(j, k, l)| \leq C B(m)$ for $2^{m}>M$. The same argument applies to the other terms in $A(m)$, which completes the proof for the first assertion.

The proof of (2.3) is essentially the same. We consider

$$
\sup _{j, l}\left\{\sum_{k \in J}(|c(j, k, l)|+|c(j, l, k)|)\right\} .
$$

It is bounded if $J=\{k:|k-l| \leq M\}$; and it is $\leq C B(m)$ if $J=\left\{k: 2^{m} \leq|k-l|<\right.$ $\left.2^{m+1}\right\}$ if $2^{m+1}>M$. This implies (2.3).

\section{3. $T$ (1) Theorem on Besov spaces}

In view of Proposition 2.2 and Proposition 2.3, we will prove the following theorem in terms of the BCR-coefficients, which implies Theorem 1.1.

THEOREM 3.1. Let $T: \mathscr{D} \longrightarrow \mathscr{D}^{\prime}$ be a Calderón-Zygmund operator with the wavelet decomposition as in (2.1), (2.2) and satisfying (1.1'), (2.3).

(i) If $\sum_{m=0}^{\infty}(m+1) A(m)<\infty$ and $\sum_{k} a(j, k, l)=\sum_{l} b(j, k, l)=0$, then $T$ is a bounded operator on $\dot{B}_{p}^{0 . q}, 1 \leq p, q<\infty$.

(ii) If $\sum_{m=0}^{\infty} 2^{\alpha m} A(m)<\infty$ and $\sum_{l} b(j, k, l)=0$ for any $j, k \in \mathbb{Z}$, then $T$ is bounded on $\dot{B}_{p}^{\alpha, q}, 0<\alpha<1,1 \leq p, q<\infty$. 
Let us rewrite

$$
T=\sum_{-\infty}^{\infty} P_{j} T Q_{j}+\sum_{-\infty}^{\infty} Q_{j} T P_{j}+\sum_{-\infty}^{\infty} Q_{j} T Q_{j}=T^{(1)}+T^{(2)}+T^{(3)}
$$

We will first consider the term $T^{(2)}$. Its distributional kernel is

$$
K^{(2)}(x, y)=\sum_{j k l} b(j, k, l) \psi_{j k}(x) \varphi_{j l}(y)
$$

Let $J_{m}=\left\{(k, l): 2^{m} \leq|k-l|<2^{m+1}\right\}$, and

$$
b_{m}(j, k, l)= \begin{cases}b(j, k, l) & (k, l) \in J_{m} \\ -\sum_{n:(k, n) \in J_{m}} b(j, k, n) & l=k \\ 0 & \text { otherwise }\end{cases}
$$

where $m=0,1,2, \ldots$ The definition implies that $\sum_{l} b_{m}(j, k, l)=0$ for each $j$, $k \in \mathbb{Z}$. Since $\sum_{l} b(j, k, l)=0$ by assumption, we have

$$
b(j, k, k)=-\sum_{m=0}^{\infty} \sum_{l:(k, l) \in J_{m}} b(j, k, l) .
$$

Hence $K^{(2)}(x, y)$ can be decomposed as

$$
\begin{aligned}
K^{(2)}(x, y)= & \sum_{j k} b(j, k, k) \psi_{j k}(x) \varphi_{j k}(y) \\
& +\sum_{j k} \sum_{l: l \neq k} b(j, k, l) \psi_{j k}(x) \varphi_{j l}(y) \\
= & \sum_{j k}\left(-\sum_{m=0}^{\infty} \sum_{l:(k, l) \in J_{m}} b(j, k, l)\right) \psi_{j k}(x) \varphi_{j k}(y) \\
& +\sum_{m=0}^{\infty} \sum_{j k} \sum_{l:(k, l) \in J_{m}} b(j, k, l) \psi_{j k}(x) \varphi_{j l}(y) \\
= & \sum_{m=0}^{\infty} \sum_{j k l} b_{m}(j, k, l) \psi_{j k}(x) \varphi_{j l}(y)=\sum_{m=0}^{\infty} K_{m}^{(2)}(x, y) .
\end{aligned}
$$

Let $T_{m}^{(2)}$ denote the operator with distributional kernel $K_{m}^{(2)}(x, y)$. Then we can decompose $T^{(2)}$ as: $T^{(2)}=\sum_{m=0}^{\infty} T_{m}^{(2)}$. We call each $T_{m}^{(2)}$ a block operator. The following lemma together with the assumption on $A(m)$ will imply that $T^{(2)}$ is a bounded operator on $\dot{B}_{p}^{\alpha, q}$. 
LEMMA 3.2. Under the hypothesis of Theorem 3.1, let $1 \leq p, q<\infty, 0 \leq \alpha<1$. Then $T_{m}^{(2)}$ is a bounded operator on $\dot{B}_{p}^{\alpha, q}$, and the operator norm satisfies

$$
\left\|T_{m}^{(2)}\right\| \leq \begin{cases}C(m+1) A(m) & \alpha=0 \\ C 2^{\alpha m} A(m) & 0<\alpha<1\end{cases}
$$

where $C$ is independent of $m$.

PROOF. Let $f(y)=\sum_{j k} \alpha(j, k) \psi_{j k}(y)$ be in $\dot{B}_{p}^{\alpha, q}$, and let $g(x)=\sum_{j k} \beta(j, k) \psi_{j k}(x)$ be in the dual space $\dot{B}_{p^{\prime}}^{-\alpha, q^{\prime}}$. Noting that $\left\langle\varphi_{j l}, \psi_{j^{\prime} k^{\prime}}\right\rangle \neq 0$ implies that $j>j^{\prime}$ and $2^{j-j^{\prime}} k^{\prime} \leq l \leq 2^{j-j^{\prime}}\left(k^{\prime}+M\right)+M$ (recall that $\varphi, \psi$ have compact supports contained in $\left.\left[0, M^{\prime}\right]\right)$, one can write

$$
\begin{aligned}
\left\langle T_{m}^{(2)} f, g\right\rangle= & \sum_{j^{\prime} k^{\prime}} \sum_{j k l} \alpha\left(j^{\prime}, k^{\prime}\right) b_{m}(j, k, l) \beta(j, k)\left\langle\varphi_{j l}, \psi_{j^{\prime} k^{\prime}}\right\rangle \\
= & \sum_{j, j^{\prime}: 0<j-j^{\prime} \leq m}\left(\sum_{k k^{\prime} l} \alpha\left(j^{\prime}, k^{\prime}\right) b_{m}(j, k, l) \beta(j, k)\left\langle\varphi_{j l}, \psi_{j^{\prime} k^{\prime}}\right\rangle\right) \\
& +\sum_{j, j^{\prime} j-j-j^{\prime} \geq m+1}\left(\sum_{k k^{\prime} l} \alpha\left(j^{\prime}, k^{\prime}\right) b_{m}(j, k, l) \beta(j, k)\left\langle\varphi_{j l}, \psi_{j^{\prime} k^{\prime}}\right\rangle\right) \\
= & \mathrm{I}+\mathrm{II} .
\end{aligned}
$$

Let $\Gamma_{k k^{\prime} l}^{m j^{\prime} s}=b_{m}\left(j^{\prime}+s, k, l\right)\left\langle\varphi_{j^{\prime}+s l}, \psi_{j^{\prime} k^{\prime}}\right\rangle$. By using Proposition 2.1 and the Hölder inequality, we obtain

$$
\begin{aligned}
& |\mathrm{I}| \leq \sum_{s=1}^{m} \sum_{j^{\prime}}\left|\sum_{k k^{\prime} l} \alpha\left(j^{\prime}, k^{\prime}\right) b_{m}\left(j^{\prime}+s, k, l\right) \beta\left(j^{\prime}+s, k\right)\left\langle\varphi_{j^{\prime}+s l}, \psi_{j^{\prime} k^{\prime}}\right\rangle\right| \\
& \leq \sum_{s=1}^{m}\left\{\sum_{j^{\prime}}\left(\sum_{k^{\prime}}\left|\alpha\left(j^{\prime}, k^{\prime}\right)\right|^{p}\right)^{1 / p}\left(\sum_{k^{\prime}}\left|\sum_{k l} \beta\left(j^{\prime}+s, k\right) \Gamma_{k k^{\prime} l}^{m j^{\prime} s}\right|^{p^{\prime}}\right)^{1 / p^{\prime}}\right\} \\
& \leq C \sum_{s=1}^{m} 2^{\left(1 / p^{\prime}+\alpha-1 / 2\right) s}\|f\|_{\dot{B}_{p}^{a,{ }^{a}}} \\
& \times\left\{\sum_{j^{\prime}}\left(\sum_{k^{\prime}}\left|2^{\left(-1 / p^{\prime}-\alpha+1 / 2\right)\left(j^{\prime}+s\right)} \sum_{k l} \beta\left(j^{\prime}+s, k\right) \Gamma_{k k^{\prime \prime l}}^{m j^{\prime} l}\right|^{p^{\prime}}\right)^{q^{\prime} / p^{\prime}}\right\}^{1 / q^{\prime}} .
\end{aligned}
$$


We will make a separate estimation of $\sum_{k^{\prime}} \cdots$. Note that

$$
\begin{aligned}
\sum_{k l}\left|\Gamma_{k k^{\prime} l}^{m j^{\prime} s}\right| & \leq 2^{-s / 2} \sum_{l} \sum_{k}\left|b_{m}\left(j^{\prime}+s, k, l\right)\left\langle\varphi(x-l), \psi\left(2^{-s} x-k^{\prime}\right)\right\rangle\right| \\
& \leq 2^{-s / 2} A(m) \sum_{l}\left|\left\langle\varphi(x-l), \psi\left(2^{-s} x-k^{\prime}\right)\right\rangle\right| \\
& \leq C 2^{s / 2} A(m),
\end{aligned}
$$

(the last inequality holds because $\varphi$ has compact support and $\sum_{l}|\varphi(x-l)|$ is bounded) and

$$
\begin{aligned}
\sum_{k^{\prime} l}\left|\Gamma_{k k^{\prime} l}^{m j^{\prime}}\right| & \leq 2^{-s / 2} \sum_{l}\left|b_{m}\left(j^{\prime}+s, k, l\right)\right|\left\langle|\varphi(x-l)|, \sum_{k^{\prime}}\left|\psi\left(2^{-s} x-k^{\prime}\right)\right|\right\rangle \\
& \leq C 2^{-s / 2} A(m) .
\end{aligned}
$$

Hence

$$
\begin{aligned}
& \sum_{k^{\prime}}\left|\sum_{k l} \beta\left(j^{\prime}+s, k\right) \Gamma_{k k^{\prime} l}^{m j^{\prime} l}\right|^{p^{\prime}} \\
& \quad \leq \sum_{k^{\prime}}\left\{\left(\sum_{k l}\left|\beta\left(j^{\prime}+s, k\right)\right|^{p^{\prime}}\left|\Gamma_{k k^{\prime} l}^{m j^{\prime} l}\right|\right)\left(\sum_{k l}\left|\Gamma_{k k^{\prime} l}^{m j^{\prime} l}\right|\right)^{p^{\prime} / p}\right\} \\
& \quad \leq C\left(\sum_{k}\left|\beta\left(j^{\prime}+s, k\right)\right|^{p^{\prime}} \sum_{k^{\prime} l}\left|\Gamma_{k k^{\prime} l}^{m j^{\prime} l}\right|\right)\left(2^{s / 2} A(m)\right)^{p^{\prime} / p} \\
& \quad \leq C\left(\sum_{k}\left|\beta\left(j^{\prime}+s, k\right)\right|^{p^{\prime}}\right)\left(2^{-s / 2} A(m)\right)\left(2^{s / 2} A(m)\right)^{p^{\prime} / p} \\
& \quad=C 2^{s\left(p^{\prime} / p-1\right) / 2}|A(m)|^{p^{\prime}} \sum_{k}\left|\beta\left(j^{\prime}+s, k\right)\right|^{p^{\prime}} .
\end{aligned}
$$

It follows that

$$
\begin{aligned}
& |\mathrm{I}| \leq C \sum_{s=1}^{m} 2^{\left(1 / p^{\prime}+\alpha-1 / 2\right) s} 2^{s\left(1 / p-1 / p^{\prime}\right) / 2} \cdot A(m)\|f\|_{\dot{B}_{p}^{\alpha, q}} \\
& \times\left\{\sum_{j^{\prime}}\left(\sum_{k}\left|2^{\left(-1 / p^{\prime}-\alpha+1 / 2\right)\left(j^{\prime}+s\right)} \beta\left(j^{\prime}+s, k\right)\right|^{p^{\prime}}\right)^{q^{\prime} / p^{\prime}}\right\}^{1 / q^{\prime}} \\
& \leq C \sum_{s=1}^{m} 2^{\alpha m} A(m)\|f\|_{\dot{B}_{i}^{\mu, q}}\|g\|_{\dot{B}_{p^{\prime}}^{-\alpha, q^{\prime}}} \\
& \leq \begin{cases}C(m+1) A(m)\|f\|_{\dot{B}_{p}^{\alpha, q}}\|g\|_{\dot{B}_{p^{\prime}}^{-\alpha, q^{\prime}}} & \alpha=0 ; \\
C 2^{\alpha m} A(m)\|f\|_{\dot{B}_{p}^{\alpha, q u}}\|g\|_{\dot{B}_{p^{\prime}}^{-\alpha, q^{\prime}}} & 0<\alpha<1 .\end{cases}
\end{aligned}
$$


We now estimate the expression II. For convenience, we use the same notation $C$ to denote the different constants in the different place. Define

$$
g_{j k}(x)=\int_{-\infty}^{x} \sum_{l} b_{m}(j, k, l) \varphi(y-l) d y \quad \text { and } \quad \tilde{\Gamma}_{k k^{\prime}}^{j^{\prime} s}=\left\langle g_{j^{\prime}+s k}(x), \psi^{\prime}\left(2^{-s} x-k\right)\right\rangle
$$

Then

$$
\begin{aligned}
|\mathbf{I I}|= & \mid \sum_{j, j^{\prime} \cdot j-j^{\prime} \geq m+1} \sum_{k k^{\prime}} \alpha\left(j^{\prime}, k^{\prime}\right) b_{m}(j, k, l) \beta(j, k)\left\langle\varphi_{j l}, \psi_{j^{\prime} k^{\prime}}||\right. \\
= & C \sum_{s=m+1}^{\infty} \sum_{j^{\prime}=-\infty}^{\infty} \sum_{k k^{\prime}} 2^{-3 . s / 2}\left|\alpha\left(j^{\prime}, k^{\prime}\right) \beta\left(j^{\prime}+s, k\right) \tilde{\Gamma}_{k k^{\prime}}^{j^{\prime} s}\right| \\
\leq & C \sum_{s=m+1}^{\infty} 2^{-3 s / 2}\|f\|_{\dot{B}_{v}^{\alpha, q}} \\
& \times\left\{\sum_{j^{\prime}} 2^{\left(-1 / p^{\prime}-\alpha+1 / 2\right) j^{\prime} q^{\prime}}\left(\sum_{k^{\prime}}\left|\sum_{k} \beta\left(j^{\prime}+s, k\right) \tilde{\Gamma}_{k k^{\prime}}^{j s^{\prime}}\right|^{p^{\prime}}\right)^{q^{\prime} / p^{\prime}}\right\}^{1 / q^{\prime}}
\end{aligned}
$$

We claim that

$$
\sum_{k^{\prime}}\left|\sum_{k} \beta\left(j^{\prime}+s, k\right) \tilde{\Gamma}_{k k^{\prime}}^{j^{\prime} s}\right|^{p^{\prime}} \leq C 2^{(s+m)\left(p^{\prime}-1\right)} 2^{m}|A(m)|^{p^{\prime}} \sum_{k}\left|\beta\left(j^{\prime}+s, k\right)\right|^{p^{\prime}}
$$

In fact, the condition $\sum_{l} b_{m}(j, k, l)=0$ implies that supp $g_{j k} \subseteq\left[k-2^{m+1}, k+2^{m+1} M\right]$. Then

$$
\begin{aligned}
\sum_{k^{\prime}}\left|\tilde{\Gamma}_{k k^{\prime}}^{j^{\prime} s}\right| & \leq C\left\langle\left|g_{j^{\prime}+s k}(x)\right|, \sum_{k^{\prime}}\left|\psi^{\prime}\left(2^{-s} x-k^{\prime}\right)\right|\right\rangle \\
& \leq C\left\langle\left|g_{j^{\prime}+s k}(x)\right|, 1\right\rangle \leq C 2^{m} A(m) .
\end{aligned}
$$

On the other hand, for $s=j-j^{\prime}$ since $\operatorname{supp} \psi^{\prime}\left(2^{-s} x-k^{\prime}\right) \subseteq\left[2^{\prime} k^{\prime}, 2^{s}\left(k^{\prime}+M\right)\right]$, we know that $\left|k-2^{s} k^{\prime}\right| \leq 2^{s} M+2^{m}+M \leq C 2^{s}$. It follows that

$$
\sum_{k}\left|\tilde{\Gamma}_{k k^{\prime}}^{j^{\prime} s}\right| \leq C \sum_{k} \| g_{j^{\prime}+s k}(x)|,| \psi^{\prime}\left(2^{-s} x-k^{\prime}\right)|\rangle \leq C 2^{s+m} A(m)
$$


Combining these two estimates we have

$$
\begin{aligned}
& \sum_{k^{\prime}}\left|\sum_{k} \beta\left(j^{\prime}+s, k\right) \tilde{\Gamma}_{k k^{\prime}}^{j^{\prime} s}\right|^{p^{\prime}} \\
& \quad \leq \sum_{k^{\prime}}\left(\sum_{k}\left|\beta\left(j^{\prime}+s, k\right)\right|^{p^{\prime}}\left|\tilde{\Gamma}_{k k^{\prime}}^{j^{\prime} s}\right|\right)\left(\sum_{k}\left|\tilde{\Gamma}_{k k^{\prime}}^{j^{\prime} s}\right|\right)^{p^{\prime} / p} \\
& \leq C 2^{(s+m)\left(p^{\prime}-1\right)} 2^{m}|A(m)|^{p^{\prime}} \sum_{k}\left|\beta\left(j^{\prime}+s, k\right)\right|^{p^{\prime}}
\end{aligned}
$$

This proves the claim. We return to the estimate of $|\mathrm{II}|$

$$
\begin{aligned}
|\mathrm{II}| \leq & C \sum_{s=m+1}^{\infty} 2^{\left(1 / p^{\prime}+\alpha-2\right) s}\|f\|_{\dot{B}_{p^{\alpha}}^{\alpha . q}}\left\{\sum _ { j ^ { \prime } } \left(2^{(s+m)\left(p^{\prime}-1\right)} 2^{m}|A(m)|^{p^{\prime}}\right.\right. \\
& \left.\left.\times \sum_{k}\left|2^{\left(-1 / p^{\prime}-\alpha+1 / 2\right)\left(j^{\prime}+s\right)} \beta\left(j^{\prime}+s, k\right)\right|^{p^{\prime}}\right)^{q^{\prime} / p^{\prime}}\right\}^{1 / q^{\prime}} \\
\leq & C\left(\sum_{s=m+1}^{\infty} 2^{(\alpha-1) s} 2^{m} A(m)\right)\|f\|_{\dot{B}_{p}^{\alpha . q}}\|g\|_{\dot{B}_{p^{\prime}}^{-\alpha, q^{\prime}}} \\
\leq & C 2^{\alpha m} A(m)\|f\|_{\dot{B}_{p}^{\alpha . q}}\|g\|_{\dot{B}_{p^{\prime}}^{-\alpha, q^{\prime}}} .
\end{aligned}
$$

By the estimates of $|\mathrm{I}|$ and $|\mathrm{II}|$ we conclude that $T^{(2)}$ is a bounded operator on $\dot{B}_{p}^{\alpha, q}$, $1 \leq p, q<\infty, 0 \leq \alpha<1$ with the operator norm as specified.

LEMMA 3.3. Under the hypothesis of Theorem 3.1, let $1 \leq p, q<\infty, 0 \leq \alpha<1$. Then $T^{(1)}$ is a bounded operator on $\dot{B}_{p}^{\alpha, q}$.

Proof. We can write

$$
\left\langle T^{(1)} f, g\right\rangle=\sum_{j j^{\prime} k k^{\prime}} a(j, k, l) \beta\left(j^{\prime}, k^{\prime}\right) \alpha(j, l)\left\langle\varphi_{j k}, \psi_{j^{\prime} k^{\prime}}\right\rangle
$$

with $j>j^{\prime}$ and $2^{j-j^{\prime}} k^{\prime} \leq l \leq 2^{j-j^{\prime}}\left(k^{\prime}+M\right)+M$ as in Lemma 3.2. For the case $\alpha=0$, we have by assumption that $\sum_{k} a(j, k, l)=0$, and we can apply the same proof as in Lemma 3.2 (by replacing $\sum_{l} b(j, k, l)=0$ ).

For the case $0<\alpha<1$, we have not assumed that $\sum_{k} a(j, k, l)=0$ and we need 
to modify the proof by separating out the diagonal term (as in (3.2)):

$$
\begin{aligned}
\left|\left\langle T^{(1)} f, g\right\rangle\right| \leq & \left|\sum_{j, j^{\prime}, j-j^{\prime}>0} \sum_{k k^{\prime}} \beta\left(j^{\prime}, k^{\prime}\right) a(j, k, k) \alpha(j, k)\left\langle\varphi_{j k}, \psi_{j^{\prime} k^{\prime}}\right\rangle\right| \\
& +\left|\sum_{m=0}^{\infty} \sum_{j, j^{\prime}, j-j^{\prime}>0} \sum_{(k, l) \in J_{m}} \sum_{k^{\prime}} \beta\left(j^{\prime}, k^{\prime}\right) a(j, k, l) \alpha(j, l)\left\langle\varphi_{j k}, \psi_{j^{\prime} k^{\prime}}\right\rangle\right| .
\end{aligned}
$$

By using the same argument as in Lemma 3.2, we can show that the first term is bounded by $C \sum_{s=1}^{\infty} 2^{-\alpha s}\|f\|_{\dot{B}_{p}^{\alpha . q}}\|g\|_{\dot{B}_{p,}^{-\alpha, q^{\prime}}}$ and the second term is bounded by $C A(m) \sum_{s=1}^{\infty} 2^{-\alpha s}\|f\|_{\dot{B}_{p}^{\alpha ., s}}\|g\|_{\dot{B}_{p^{\prime}}^{-\alpha . q^{\prime}}}$ (note that in here the term $\sum_{s=1}^{\infty} 2^{-\alpha s}$ converges and we only need to use the estimation for $|\mathrm{I}|$ without recoursing to the estimation $|\mathrm{II}|$ in the last proof). This proves the lemma.

LEMMA 3.4. Under the hypothesis of Theorem 3.1 let $1 \leq p, q<\infty, 0 \leq \alpha<1$. Then $T^{(3)}$ is a bounded operator on the Besov spaces $\dot{B}_{p}^{\alpha, q}$.

ProOF. We can write

$$
\left\langle T^{(3)} f, g\right\rangle=\sum_{j k l} c(j, k, l) \alpha(j, k) \beta(j, l),
$$

where $f, g$ are defined as in Lemma 3.2. By using the duality and condition (2.3), it can be checked as in Lemma 3.2 that $\left|\left\langle T^{(3)} f, g\right\rangle\right| \leq C\|f\|\left\|_{\dot{B}_{\nu^{\prime . q}}^{\alpha . q}}\right\| g \|_{\dot{B}_{p^{\prime}}^{-\alpha . q^{\prime}}}$.

Theorem 3.1 follows directly from Lemmas 3.2-3.4.

\section{4. $T$ (1) Theorem on Triebel-Lizorkin spaces}

In view of Proposition 2.2 and Proposition 2.3, we will prove the following theorem in terms of the BCR-coefficients, which implies Theorem 1.2.

THEOREM 4.1. Let $T: \mathscr{D} \longrightarrow \mathscr{D}^{\prime}$ be a Calderón-Zygmund operator with the wavelet decomposition as in (2.1), (2.2) and satisfying $\left(1.1^{\prime}\right),(2.3)$.

(i) If $\sum_{m=0}^{\infty}(m+1)^{2-1 / q} A(m)<\infty$, and $\sum_{k} a(j, k, l)=\sum_{l} b(j, k, l)=0$, then $T$ is a bounded operator on $\dot{F}_{p}^{0, q}, 1 \leq p, q<\infty$.

(ii) If $\sum_{m=0}^{\infty} 2^{\alpha m} A(m)<\infty$ and $\sum_{l} b(j, k, l)=0$ for any $j, k \in \mathbb{Z}$, then $T$ is bounded on $\dot{F}_{p}^{\alpha . q}, 0<\alpha<1,1 \leq p, q<\infty$.

We will first prove the theorem for $\dot{F}_{1}^{\alpha, q}$, then apply an interpolation theorem 
on $\dot{F}_{1}^{\alpha, q}$ and $\dot{F}_{q}^{\alpha, q}\left(=\dot{B}_{q}^{\alpha, q}\right)$, and a duality argument to conclude the theorem. We need the following notion of atom which can be found in [9].

DEFINITION. Let $a(x)=\sum_{j k} \alpha(j, k) \psi_{j k}(x)$ be a locally integrable function. We say that $a(x)$ is an $(\alpha, 1, q)$-atom if there is a dyadic cube $l \subset \mathbb{R}$ such that

(i) $\operatorname{supp} a(x) \subset I$;

(ii) $\int_{1} a(x) d x=0$;

(iii) $\|A(a)\|_{q} \leq|I|^{1 / q-1}$,

where $A(a)$ is defined by

$$
A(a)(x)=\left(\sum_{j, k} 2^{(\alpha+1 / 2) j q}|\alpha(j, k)|^{q} \chi\left(2^{j} x-k\right)\right)^{1 / q}
$$

For $j, k \in \mathbb{Z}$, let $l_{j k}$ denote the interval $\left[2^{-j} k, 2^{-j}(k+1)\right]$. Let

$$
a_{j k}(x)=\sum_{j^{\prime}, k^{\prime}: I_{j^{\prime} k^{\prime}} \subset I_{j k}} \alpha\left(j^{\prime}, k^{\prime}\right) \psi_{j^{\prime} k^{\prime}}(x)
$$

Note that the sum is actually adding all $j^{\prime}, k^{\prime}$ with $j \leq j^{\prime}, k^{\prime} \in\left[2^{j^{\prime}-j} k, 2^{j^{\prime}-j}(k+1)\right)$. It follows that the support of $a_{j k}(x)$ is contained in $\left[2^{-j} k, 2^{-j}(k+M)\right]$. By the definition, we know that $a_{j k}(x)$ is an $(\alpha, 1, q)$-atom if

$$
\left\|a_{j k}\right\|_{(\alpha, 1, q)}:=\left\{2^{-j(q-1)} \sum_{j^{\prime}, k^{\prime}: I_{j^{\prime} k^{\prime}} \subset l_{j k}} 2^{(-1 / q+\alpha+1 / 2) j^{\prime} q}\left|\alpha\left(j^{\prime}, k^{\prime}\right)\right|^{q}\right\}^{1 / q}<\infty
$$

LEMMA 4.2. Let $a_{j k}(x)$ be the atom as in (4.2), and let $T_{m}^{(2)}$ be defined as in Lemma 3.2. Then we have

$$
\left\|T_{m}^{(2)} a_{j k}\right\|_{\dot{F}_{1}^{u, q}} \leq \begin{cases}C(m+1)^{2-1 / q} A(m)\left\|a_{j k}\right\|_{(\alpha, 1, q)} & \alpha=0 \\ C 2^{\alpha m} A(m)\left\|a_{j k}\right\|_{(\alpha, 1, q)} & 0<\alpha<1\end{cases}
$$

where $C$ is independent of $m, j, k$.

PROOF. Without loss of generality we consider $a_{00}(y)$ and denote it by $a(y)$ for simplicity, that is, $a(y)=\sum_{0 \leq j} \sum_{0 \leq k<2} \alpha(j, k) \psi_{j k}(y)$. Noting that $\left\langle\varphi_{j l}, \psi_{j^{\prime} k^{\prime}}\right\rangle \neq 0$ 
implies that $j>j^{\prime}$ and $2^{j-j^{\prime}} k^{\prime} \leq l \leq 2^{j-j^{\prime}}\left(k^{\prime}+M\right)+M$. One can write

$$
\begin{aligned}
\left(T_{m}^{(2)} a\right)(x)= & \sum_{j k l} \sum_{j^{\prime} k^{\prime}} \alpha\left(j^{\prime}, k^{\prime}\right) b_{m}(j, k, l)\left\langle\varphi_{j l}, \psi_{j^{\prime} k^{\prime}}\right\rangle \psi_{j k}(x) \\
= & \sum_{j \leq m}\left(\sum_{j^{\prime} k k^{\prime} l} \alpha\left(j^{\prime}, k^{\prime}\right) b_{m}(j, k, l)\left\langle\varphi_{j l}, \psi_{j^{\prime} k^{\prime}}\right\rangle \psi_{j k}(x)\right) \\
& +\sum_{j>m}\left(\sum_{j^{\prime}: 0<j-j^{\prime} \leq m} \sum_{k k^{\prime} l} \alpha\left(j^{\prime}, k^{\prime}\right) b_{m}(j, k, l)\left\langle\varphi_{j l}, \psi_{j^{\prime} k^{\prime}}\right\rangle \psi_{j k}(x)\right) \\
& +\sum_{j>m}\left(\sum_{j^{\prime}: j-j^{\prime}>m} \sum_{k k^{\prime} l} \alpha\left(j^{\prime}, k^{\prime}\right) b_{m}(j, k, l)\left\langle\varphi_{j l}, \psi_{j^{\prime} k^{\prime}}\right\rangle \psi_{j k}(x)\right) \\
= & a_{1}(x)+a_{2}(x)+a_{3}(x) .
\end{aligned}
$$

Since $\dot{B}_{1}^{\alpha, 1}=\dot{F}_{1}^{\alpha, 1} \subset \dot{F}_{1}^{\alpha, q}, 1 \leq q<\infty$, it follows that there exists a constant $C$ such that $\|f\|_{\dot{F}_{1}^{\alpha .4}} \leq C\|f\|_{\dot{B}_{1}^{\alpha .1}}$. Using Proposition 2.1 and the Hölder inequality, we obtain

$$
\begin{aligned}
& \left\|a_{1}\right\|_{\dot{F}_{1}^{a . q}} \leq C\left\|\sum_{j \leq m} \sum_{j^{\prime} k k^{\prime} l} \alpha\left(j^{\prime}, k^{\prime}\right) b_{m}(j, k, l)\left\langle\varphi_{j l}, \psi_{j^{\prime} k^{\prime}}\right\rangle \psi_{j k}\right\|_{\dot{B}_{1}^{\alpha, l}} \\
& \leq C \sum_{j \leq m} \sum_{j^{\prime} k k^{\prime} l} 2^{(\alpha-1 / 2) j}\left|\alpha\left(j^{\prime}, k^{\prime}\right) b_{m}(j, k, l)\left\langle\varphi_{j l}, \psi_{j^{\prime} k^{\prime}}\right\rangle\right| \\
& \leq C A(m) \sum_{0 \leq j \leq m} \sum_{0<j^{\prime}<j} \sum_{0 \leq k^{\prime}<2^{\prime}} 2^{\left(j-j^{\prime}\right) / 2} 2^{(\alpha-1 / 2) j}\left|\alpha\left(j^{\prime}, k^{\prime}\right)\right| \\
& \leq C A(m)\left\{\sum_{0 \leq j \leq m} 2^{\alpha j}\left(\sum_{0<j^{\prime}<m} 2^{-\alpha j^{\prime} q^{\prime}}\right)^{1 / q^{\prime}}\right\} \\
& \times\left(\sum_{j^{\prime} k^{\prime}} 2^{(-1 / q+\alpha+1 / 2) j^{\prime} q}\left|\alpha\left(j^{\prime}, k^{\prime}\right)\right|^{q}\right)^{1 / q} \\
& \leq \begin{cases}C(m+1)^{2-1 / q} A(m)\|a\|_{(\alpha, 1, q)} & \alpha=0 ; \\
C 2^{\alpha m} A(m)\|a\|_{(\alpha, 1, q)} & 0<\alpha<1 .\end{cases}
\end{aligned}
$$

To estimate $\left\|a_{2}\right\|_{F_{1}^{u . q}}$, we first observe that the condition $0 \leq k^{\prime}<2^{j^{\prime}}$ implies that $0 \leq 2^{-j^{\prime}} l \leq M$. By the expression of $a_{2}(x)$, we know that $k \in E_{j}=$ $\left[-2^{m+1}, 2^{m+1}+2^{j}+2^{j} M\right]$, and hence

$$
\operatorname{supp} a_{2}(x) \subseteq \bigcup_{j>m} \bigcup_{k \in E_{j}}\left[2^{-j} k, 2^{-j}(k+M)\right] \subseteq[-1,2 M]
$$


Let $\Gamma_{k k^{\prime} l}^{m j s}=b_{m}(j, k, l)\left\langle\varphi_{j l}, \psi_{j-s k^{\prime}}\right\rangle$. Then

$$
\begin{aligned}
\left\|a_{2}\right\|_{\dot{F}_{1}^{\alpha, q}} & \leq C\left\|\sum_{j>m} \sum_{j^{\prime}: 0<j-j^{\prime} \leq m} \sum_{k k^{\prime} l} \alpha\left(j^{\prime}, k^{\prime}\right) b_{m}(j, k, l)\left(\varphi_{j l}, \psi_{j^{\prime} k^{\prime}}\right\rangle \psi_{j k}(x)\right\|_{\dot{F}_{1}^{\alpha . q}} \\
& \left.\leq C \sum_{s=1}^{m}\left\|\sum_{j=m+1}^{\infty} \sum_{k l} \sum_{0 \leq k^{\prime}<j^{-s}} \alpha\left(j-s, k^{\prime}\right) \Gamma_{k k^{\prime} l}^{m j s} \psi_{j k}\right\|_{\dot{F}_{1}^{\alpha, q}}\left|\sum_{k^{\prime} l} \alpha\left(j-s, k^{\prime}\right) \Gamma_{k k^{\prime} l}^{m j s}\right|^{q} \chi\left(2^{j} x-k\right)\right)^{1 / q} d x \\
& \leq C \sum_{s=1}^{m} \int\left(\sum_{\mathbf{R}}^{\infty} \sum_{i=m+1} 2_{k}^{(\alpha+1 / 2) j q} \mid \sum_{s=1}^{m}\left\{\sum_{j=m+1}^{\infty} \sum_{k} 2^{-j} 2^{(\alpha+1 / 2) j q}\left|\sum_{k^{\prime} l} \alpha\left(j-s, k^{\prime}\right) \Gamma_{k k^{\prime} l}^{m j}\right|^{q}\right\}^{1 / q} .\right.
\end{aligned}
$$

Similar to the estimate (3.3) in Lemma 3.2, we have

$$
\sum_{k}\left|\sum_{k^{\prime} l} \alpha\left(j-s, k^{\prime}\right) \Gamma_{k k^{\prime} l}^{m j s}\right|^{q} \leq C 2^{s\left(1-q / q^{\prime}\right) / 2}|A(m)|^{q} \sum_{k^{\prime}}\left|\alpha\left(j-s, k^{\prime}\right)\right|^{q} .
$$

It follows that

$$
\begin{aligned}
\left\|a_{2}\right\|_{\dot{F}_{1}^{\alpha, q}} & \leq C A(m) \sum_{s=1}^{m}\left\{\sum_{j=m+1}^{\infty} 2^{-j+(\alpha+1 / 2) j q} 2^{s\left(1-q / q^{\prime}\right) / 2} \sum_{k^{\prime}}\left|\alpha\left(j-s, k^{\prime}\right)\right|^{q}\right\}^{1 / q} \\
& \leq C A(m) \sum_{s=1}^{m} 2^{\alpha s}\left\{\sum_{j=m+1}^{\infty} \sum_{k^{\prime}} 2^{(-1 / q+\alpha+1 / 2)(j-s) q}\left|\alpha\left(j-s, k^{\prime}\right)\right|^{q}\right\}^{1 / q} \\
& \leq \begin{cases}C(m+1) A(m)\|a\|_{(\alpha, 1, q)} & \alpha=0 ; \\
C 2^{\alpha m} A(m)\|a\|_{(\alpha, 1, q)} & 0<\alpha<1 .\end{cases}
\end{aligned}
$$

We now turn to estimate the term $a_{3}(x)$. Like $a_{2}(x)$, supp $a_{3}(x) \subseteq[-1,2 M]$. Let $g_{j k}(x)$ be defined as in the estimate of $|\mathrm{II}|$ of Lemma 3.2 and $\tilde{\Gamma}_{k k^{\prime}}^{j s}=\left\langle g_{j k}(x)\right.$, $\left.\psi^{\prime}\left(2^{-s} x-k^{\prime}\right)\right\rangle$. Then

$$
\begin{aligned}
\left\|a_{3}(x)\right\|_{\dot{F}_{1}^{\alpha . q}} & \leq C\left\|\sum_{j>m} \sum_{j^{\prime}: j-j^{\prime}>m} \sum_{k k^{\prime} l} \alpha\left(j^{\prime}, k^{\prime}\right) b_{m}(j, k, l)\left\langle\varphi_{j l}, \psi_{j^{\prime} k^{\prime}}\right\rangle \psi_{j k}(x)\right\|_{\dot{F}_{1}^{\alpha . q}} \\
& \leq C \sum_{s=m+1}^{\infty} 2^{-3 s / 2}\left\|\sum_{j=m+1}^{\infty} \sum_{k k^{\prime}} \alpha\left(j-s, k^{\prime}\right) \tilde{\Gamma}_{k k^{\prime}}^{j s} \psi_{j k}(x)\right\| \|_{\dot{F}_{1}^{\alpha, q}} \\
& \leq C \sum_{s=m+1}^{\infty} 2^{-3 s / 2}\left\{\sum_{j=m+1}^{\infty} \sum_{k} 2^{-j+(\alpha+1 / 2) j q}\left|\sum_{k^{\prime}} \alpha\left(j-s, k^{\prime}\right) \tilde{\Gamma}_{k k^{\prime}}^{j s}\right|^{q}\right\}^{1 / q} .
\end{aligned}
$$


By the same estimate as (3.4) in Lemma 3.2, we obtain

$$
\sum_{k}\left|\sum_{k^{\prime}} \alpha\left(j-s, k^{\prime}\right) \tilde{\Gamma}_{k k^{\prime}}^{j s}\right|^{q} \leq C 2^{s+m} 2^{m q / q^{\prime}}|A(m)|^{q} \sum_{k^{\prime}}\left|\alpha\left(j-s, k^{\prime}\right)\right|^{q} .
$$

Hence

$$
\begin{aligned}
\left\|a_{3}\right\|_{\dot{F}_{1}^{\alpha, q}} & \leq C \sum_{s=m+1}^{\infty} 2^{-3 s / 2}\left\{\sum_{j=m+1}^{\infty} 2^{-j+(\alpha+1 / 2) j q} 2^{s+m} 2^{m q / q^{\prime}} \sum_{k^{\prime}}\left|\alpha\left(j-s, k^{\prime}\right)\right|^{q}\right\}^{1 / q} \\
& \leq C A(m) \sum_{s=m+1}^{\infty} 2^{m} 2^{(\alpha-1) s}\left\{\sum_{j k^{\prime}} 2^{(-1 / q+\alpha+1 / 2)(j-s) q}\left|\alpha\left(j-s, k^{\prime}\right)\right|^{q}\right\}^{1 / q} \\
& \leq C 2^{\alpha m} A(m)\|a\|_{(\alpha, 1, q)}
\end{aligned}
$$

We have hence proved Lemma 4.2 by combining the estimates of $a_{1}(x), a_{2}(x)$ and $a_{3}(x)$.

Now we state the following atomic decomposition of $\dot{F}_{1}^{\alpha, q}$, which is given in [1] for $\dot{F}_{1}^{0,2}\left(=H^{1}\right)$ and in [6] and [9] for the general case. For completeness we modify their proof and sketch it here.

LEMMA 4.3. Let $f(x)=\sum_{j k} \alpha(j, k) \psi_{j k}(x)$ be in $\dot{F}_{1}^{\alpha, q}$. Then there exists a sequence $\left\{h_{s n}(x)\right\}_{s, n}$ of $(\alpha, 1, q)$-atoms and $\left\{\lambda_{s n}\right\} \in \mathbb{R}$ such that

$$
f(x)=\sum_{s, n} \lambda_{s, n} h_{s n}(x) \quad \text { and } \quad C_{1}\|f\|_{\dot{F}_{1}^{\alpha, q}} \leq \sum_{s, n}\left|\lambda_{s n}\right| \leq C_{2}\|f\|_{\dot{F}_{1}^{\alpha, q}}
$$

for some fixed $C_{1}, C_{2}>0$ independent of $f$.

ProOF. By Proposition 2.1, $f \in \dot{F}_{1}^{\alpha, q}$ has an equivalent norm given by $\|f\|_{\dot{F}_{1}^{u, q}} \approx\|A(f)\|_{1}$.

If $2^{s}, s \in \mathbb{Z}$, is a given threshold, we define $\Omega_{s}=\left\{x: A(f)(x)>2^{s}\right\}$. This allows us to write $\Omega_{s}=\bigcup_{n \in \mathbb{N}} Q_{s n}$, where each $Q_{s n}$ is a maximal dyadic interval in $\Omega_{s}$. The intervals $Q_{s n}$, being dyadic and maximal, are either identical or disjoint. For each $s \in \mathbb{Z}, n \in \mathbb{N}$, consider the family $\mathscr{F}_{s n}$ of all dyadic intervals $I_{j k}$ such that $I_{j k} \subset Q_{s n}$ which are contained in no $Q_{s+1 p}$ for any $p$. By the above construction, we can write

$$
\bigcup_{s} \Omega_{s}=\bigcup_{s, n}\left(\bigcup_{I_{j k} \in \mathcal{F}_{s n}} I_{j k}\right)
$$


For each $s \in \mathbb{Z}, n \in \mathbb{N}$, let

$$
h_{s n}(x)=\left|\lambda_{s n}\right|^{-1} \sum_{l_{j k} \in \mathscr{F}_{s n}} \alpha(j, k) \psi_{j k}(x),
$$

where $\lambda_{s n}=\left|Q_{s n}\right|^{1-1 / q}\left\{\sum_{I_{j k} \in \mathscr{F}_{s n}} 2^{(-1 / q+\alpha+1 / 2) j q}|\alpha(j, k)|^{q}\right\}^{1 / q}$. Then, $h_{s n}(x)$ is an $(\alpha, 1, q)$-atom and

$$
f(x)=\sum_{j, k} \alpha(j, k) \psi_{j k}(x)=\sum_{s, n} \lambda_{s n} h_{s n}(x)
$$

gives an atomic decomposition of $f$. For the details we refer the reader to $[1,6,9]$.

ProOF OF THEOREM 4.1. By Lemma 4.4 we can write $f(x) \in \dot{F}_{1}^{\alpha, q}$ as an atomic decomposition $f(x)=\sum_{s, n} \lambda_{s n} h_{s n}(x)$, where each $h_{s n}(x)$ is an $(\alpha, 1, q)$-atom defined in (4.4). For each $h_{s n}(x)$, we can rewrite it as the form in (4.2) by assigning $\alpha(j, k)=0$ for $I_{j k} \subset Q_{s n}$ but $I_{j k} \notin \mathscr{F}_{s n}$. Using Lemma 4.2 and Lemma 4.3, we have

$$
\left\|T_{m}^{(2)} f\right\|_{\dot{F}_{1}^{\alpha, q}} \leq \begin{cases}C(m+1)^{2-1 / q} A(m) \sum_{s, n}\left|\lambda_{s n}\right| & \alpha=0 \\ C 2^{\alpha m} A(m) \sum_{s, n}\left|\lambda_{s n}\right| & 0<\alpha<1,\end{cases}
$$

where $C$ is independent of $m, s, n$. It follows that $T^{(2)}$ is bounded on $\dot{F}_{1}^{\alpha, q}$. Similarly as in Lemma 3.3, and Lemma 3.4, we can show that both $T^{(1)}$ and $T^{(3)}$ are also bounded operators on $\dot{F}_{1}^{\alpha, q}$. Hence, we have proved that $T$ is bounded on $\dot{F}_{1}^{\alpha, q}$, and

$$
\|T\|_{\left(\dot{f}_{1}^{\alpha, q}, \dot{F}_{1}^{\alpha, q}\right)} \leq \begin{cases}C \sum_{m=0}^{\infty}(m+1)^{2-1 / q} A(m) & \alpha=0 ; \\ C \sum_{m=0}^{\infty} 2^{\alpha m} A(m) & 0<\alpha<1,\end{cases}
$$

where $0 \leq \alpha<1,1 \leq q<\infty$.

Since $\bar{T}$ is bounded on $\dot{B}_{q}^{\alpha, q}\left(=\dot{F}_{q}^{\alpha, q}\right)$, the interpolation theorem [13] implies that $T$ is bounded on $\dot{F}_{p}^{\alpha, q}, 0 \leq \alpha<1,1 \leq p \leq q$. Similarly as in the proof of Theorem 3.1 and Theorem 4.1, we can show, by interchanging the role of $T(1)$ and $T^{*}(1)$, that $T$ is bounded on both $\dot{F}_{q}^{\alpha, q}$ and $\dot{F}_{1}^{\alpha, q}$ with $-1<\alpha \leq 0,1 \leq q<\infty$. Again, applying the interpolation theorem and the duality, $T$ is bounded on $\dot{F}_{p}^{\alpha, q}, 0 \leq \alpha<1, p \geq q$. This finishes the proof of Theorem 4.1 .

ProOF OF COROLlary 1.3. With the above notation, we have $\left\|T_{m}^{(2)}\right\|_{\left(H^{1}, H^{1}\right)} \leq$ $C(m+1)^{3 / 2} A(m)$ and $\left\|T_{m}^{(2)}\right\|_{\left(L^{2}, L^{2}\right)} \leq C(m+1)^{1 / 2} A(m)$ for some $C>0$ independent of $m$. For $1<p<2$, by the interpolation theorem we have $\left\|T_{m}^{(2)}\right\|_{\left(L^{p}, L^{p}\right)} \leq$ $C(m+1)^{1 / 2+2(1 / p-1 / 2)} A(m)$. Using the duality argument, for $2<p<\infty$ we have $\left\|T_{m}^{(2)}\right\|_{\left(L^{p}, L^{p}\right)} \leq C(m+1)^{1 / 2+2 \mid 1 / p-1 / 21} A(m)$. It follows from the assumption on $A(m)$ that $T^{(2)}$ is bounded on $L^{p}$. Similarly, $T^{(1)}$ and $T^{(3)}$ are bounded operators on $L^{p}$; so is $T$. This completes the proof of Corollary 1.3 . 


\section{Acknowledgement}

We thank the referee for valuable comments and suggestions.

\section{References}

[1] J. Aguirre, M. Escobedo, J. C. Perel and $\mathrm{Ph}$. Tchamitchian, 'Basis of wavelets and atomic decompositions of $H^{1}\left(\mathbb{R}^{n}\right)$ and $H^{1}\left(\mathbb{R}^{n} \times \mathbb{R}^{n}\right)$, , Proc. Amer. Math. Soc. 111 (1991), 683-693.

[2] G. Beylkin, R. Coifman and V. Rokhlin, 'Fast wavelet transforms and numerical algorithms', Comm. Pure Appl. Math. 44 (1991), 141-183.

[3] I. Daubechies, Ten lectures on wavelets, CBMS-NSF Regional Conference Series in Appl. Math. 61 (SIAM, Philadelphia, 1992).

[4] G. David and J. L. Journé, 'A boundedness criterion for generalized Calderón-Zygmund operators', Ann. of Math. 120 (1984), 371-397.

[5] D. G. Deng, L. X. Yan and Q. X. Yang, 'Blocking analysis and $T(1)$ theorem', Science in China 41 (1998), 800-808.

[6] M. Frazier, B. Jawerth and G. Weiss, Littlewood-Paley theory and the study of functions, CBMSRegional Conference Series in Mathematics 79 (AMS, Providence, RI, 1991).

[7] M. Frazier, R. Torres and G. Weiss, 'The boundedness of Calderón-Zygmund operator on the spaces $\dot{F}_{p}^{\alpha, q}$, Rev. Mat. Iberoamericana 4 (1998), 41-72.

[8] Y. Han and S. Hofmann, 'T(1) theorem for Besov and Triebel-Lizorkin spaces', Trans. Amer. Math. Soc. 237 (1993), 839-853.

[9] Y. Han, M. Paluszynski and G. Weiss, 'A new atomic decomposition for the Triebel-Lizorkin spaces', Contemporary Math. 189 (1995), 235-249.

[10] P. G. Lemarié, 'Continuité sur les espaces de Besov and operatéurs definis par des intégrales singulières', Ann. Inst. Fourier (Grenoble) 35 (1985), 175-187.

[11] Y. Meyer, La minimalité de l'espace de Besov $\dot{B}_{1}^{01}$ et la continuité des opérateurs definis par des integrales singulières, Monografias de Matematicas, 4 (Univ. Autonoma de Madrid, 1986).

[12] Ondelettes et opérateurs, Vols I, II (Hermann, Paris, 1990).

[13] H. Triebel, Theory of function spaces (Birkhäuser, Basel, 1983).

Department of Mathematics

The Chinese University of Hong Kong

Shatin, NT, Hong Kong

e-mail:kslau@math.cuhk.edu.hk
Department of Mathematics

Zhongshan University Guangzhou, 510275

P. R. China

e-mail: lixin@ics.mq.edu.au 